\title{
Intra-Abdominal Paraganglioma
}

National Cancer Institute

\section{Source}

National Cancer Institute. Intra-Abdominal Paraganglioma. NCI Thesaurus. Code C5326.

A benign or malignant extra-adrenal paragang lioma arising from the abdominal cavity. 